\title{
Perceived coercion, therapeutic relationships, and outcomes in community treatment Beth Angell
}

\author{
Address: University of Chicago, 969 E. 60th St., Chicago, Illinois 60637, USA \\ from WPA Thematic Conference. Coercive Treatment in Psychiatry: A Comprehensive Review \\ Dresden, Germany. 6-8 June 2007 \\ Published: 19 December 2007 \\ BMC Psychiatry 2007, 7(Suppl I):S55 doi:I0.1 186/I47|-244X-7-SI-S55
}

This abstract is available from: http://www.biomedcentral.com//47I-244X/7/SI/S55

(c) 2007 Angell; licensee BioMed Central Ltd.

\section{Background}

A longstanding concern regarding the use of coercive interventions and treatment pressures is their potentially damaging effect on clients' engagement in treatment and the quality of the relationship between clients and clinicians. So too, it is believed that more favorable client-clinician relationships decrease the need for coercive interventions, since they signify a mutual engagement in the treatment process. However, little research assesses the directionality of these processes.

\section{Methods}

This presentation details an analysis of data from the MacArthur Prevalence Study of Mandated Treatment, a multisite study of clients with mental illness receiving services in public mental health settings in the U.S. were surveyed about their experiences of pressures to enter and adhere to treatment. In one site of the study, a cohort of 205 clients was so surveyed and re-interviewed 8 months later. Using these longitudinal data, we assess the relationship between client-reported perceived coercion, the therapeutic relationship as rated by the client, self-reported adherence, and client-reported and interviewer-rated outcomes over time using a series of structural equation models. In each model, in order to test for reciprocal effects between perceived coercion and the therapeutic relationship, we estimated models with cross-lagged (e.g., effect of time 1 coercion on time 2 relationship, and the effect of time 1 relationship on time 2 coercion) coefficients.

\section{Results}

Results suggest that the level of negativity in the clientprovider relationships associated with increased perceived coercion over time, as well as with decreases in functioning and sense of autonomy. In contrast, perceived coercion was not related to shifts in the quality of the client- provider relationship over time. However, greater treatment adherence and positive attitudes toward medication were predictive of increased quality of the client-provider relationship.

\section{Conclusion}

Implications for clinical services are discussed. 